\title{
Molecular analysis of the SMN gene mutations in spinal muscular atrophy patients in China
}

\author{
W.L. Liu ${ }^{1,2}$, F. Li ${ }^{3}$, Z.X. He ${ }^{2}$, R. $\mathrm{Ai}^{2}$ and H.W. $\mathrm{Ma}^{1}$ \\ ${ }^{1}$ Department of Developmental Pediatrics, \\ The Affiliated Shengjing Hospital of China Medical University, \\ Shenyang, China \\ ${ }^{2}$ Department of Pediatrics, Affiliated Hospital of Guiyang Medical College, \\ Guiyang, China \\ ${ }^{3}$ Department of Ophthalmology, Affiliated Hospital of Guiyang Medical College, \\ Guiyang, China \\ Corresponding authors: H.W. Ma / W.L. Liu \\ E-mail: mahongwei1960@163.com / liuweiliang205@aliyun.com
}

Genet. Mol. Res. 12 (3): 3598-3604 (2013)

Received October 8, 2012

Accepted March 15, 2013

Published September 13, 2013

DOI http://dx.doi.org/10.4238/2013.September.13.4

\begin{abstract}
Spinal muscular atrophy (SMA) is one of the most common autosomal recessive diseases. Survival motor neuron1 (SMN1) is the SMA disease-determining gene. We examined the molecular basis of SMA in 113 Chinese SMA patients. Homozygous exon 7 and 8 deletions in SMN1 were detected by PCR-RFLP. Heterozygous deletion of SMN1 was analyzed based on variation of the sequencing peak height of the two different base pairs of exons 7 and 8 between SMN1 and SMN2. Subtle mutation was detected by genomic sequencing in the patients with heterozygous deletion of $S M N 1$. In our study, the rate of deletion of $S M N 1$ exon 7 and/or 8 was $91.2 \%$; the rate of subtle mutations was $1.8 \%$. We detected the same subtle mutation (p.Leu228X) of $S M N$ exon 5 in two patients (one type I, one type III). The p.Ser8LysfsX23 and p.Leu228X mutations accounted for 13 of the 23 families with subtle mutations reported in the SMN1 gene of Chinese SMA. This is the first report where the phenotype of SMA-type III is associated with p.Leu228X. We found two subtle mutation hotspots (p.Ser8LysfsX23 and p.Leu228X) of SMN1 exons 1 and 5 in Chinese SMA patients. These two mutations have not been reported from America or
\end{abstract}


Europe. It is proposed that the distribution of subtle mutations of SMN1 of SMA is associated with ethnicity or geographic origin.

Key words: Chinese SMA; SMN gene; Deletion mutation; Subtle mutation hotspot

\section{INTRODUCTION}

Spinal muscular atrophy (SMA) is one of the most common autosomal recessive diseases affecting $1 / 6000$ to $1 / 10000$ births worldwide. The carrier frequency is $1 / 35$ (Feldkötter et al., 2002; Cusin et al., 2003). The clinical characteristics of the disease are basically caused by the progressive loss of alpha motor neurons in spinal cord anterior horn cells, which leads to lower motor neuron degeneration and weakness and wasting of voluntary muscle. Childhood onset SMA is clinically subdivided into three types based on age of onset and clinical severity. Type I SMA patients (Werding-Hoffmann disease; MIM\# 253300) present onset before 6 months after birth and usually die within two years of age. They are never able to sit or walk. Type II SMA patients (intermediate form; MIM\#253550) show onset after 6 months of age. They can sit but are never able to walk unaided. Type III SMA patients (Kugelberg-Welander disease; MIM\# 253400) show their first symptoms after 18 months. They are able to sit and walk, but often become wheelchair bound during youth or adulthood. In 1995, the survival motor neuron1 (SMN1) was identified as the SMA disease-determining gene (Lefebvre et al., 1995). In SMA, approximately $94 \%$ of individuals with clinically typical SMA lack both copies of SMN1 exon 7, approximately $4 \%$ of cases are caused either by compound heterozygosity with a subtle mutation in one allele and a deletion in the other or by compound heterozygous subtle mutations in the $S M N 1$ gene, according to the first comprehensive review of $S M N 1$ mutations (Wirth et al., 2000). SMN2 gene is a highly homologous copy of $S M N 1$, where $S M N 1$ and $S M N 2$ differ by only five nucleotides $(-45 \mathrm{G} \rightarrow \mathrm{A}$ in intron $6,+6 \mathrm{C} \rightarrow \mathrm{T}$ in exon $7,+100 \mathrm{~A} \rightarrow \mathrm{G}$ and $+214 \mathrm{~A} \rightarrow \mathrm{G}$ in intron $7,+245 \mathrm{G} \rightarrow \mathrm{A}$ in exon 8 ). SMN2 is not directly associated with SMA because the homozygous absence of $S M N 2$ is detected in $4.5 \%$ of normal individuals (Lefebvre et al., 1995), but the number of $S M N 2$ copies is recognized as a modifying factor in the phenotypic manifestations of SMA (Lefebvre et al., 1995; Wirth et al., 2000).

To date, about 60 subtle mutations have been identified in the SMN1 gene of SMA patients worldwide. The most common sites of subtle mutations were located in exons 6 and 3 in the United States and Europe (Wirth et al., 2000; Parsons et al., 1998; Alías et al., 2009). Population-based mutations of SMN1 may be different, particularly in subtle mutations. This study aimed to determine the molecular basis of SMA in a large number of Chinese patients.

\section{MATERIAL AND METHODS}

\section{Patients}

We analyzed 113 Chinese SMA patients (48 type I, 38 type II, 27 type III). All patients fulfilled diagnostic criteria for proximal SMA as defined by the International SMA Consortium (Zerrres et al., 1999). Blood samples were collected from the patients and genomic DNA was isolated from peripheral blood lymphocytes using standard procedures. We summarized subtle mutation findings of $S M N 1$ in Chinese SMA patients in previously reported subtle mutations and our study in 26 cases of 23 families. 


\section{Mutation analysis}

Homozygous exons 7 and 8 deletions in the $S M N 1$ gene were detected by PCR-restriction fragment length polymorphism (RFLP) assay (Van der Steege et al., 1995). Patients who did not show homozygous deletion of the $S M N 1$ gene (exons 7 and 8) were further analyzed for heterozygous deletion of $S M N 1$ by sequencing of $S M N$, where the heterozygous deletion of $S M N 1$ was analyzed based on the variation of the sequencing peak height of the two different base pairs of exons 7 and 8 between $S M N 1$ and $S M N 2$ (Qu et al., 2011). For analysis of non-deletional mutation of the $S M N$ gene, the entire exons and exon-intron boundaries of the $S M N$ gene were sequenced. All of the exons and exon-intron boundaries of $S M N$ were amplified by PCR according to standard literature methods with regard to the designed primers and the amplification conditions (Tsai et al., 2001). PCR was performed in a 50- $\mu \mathrm{L}$ reaction system containing $10 \mathrm{X}$ PCR buffer, $0.1 \mathrm{mM}$ dNTPs, $20 \mathrm{mM} \mathrm{Mg}{ }^{2+}, 200 \mathrm{ng}$ genomic DNA, $10 \mu \mathrm{M}$ each sense and antisense primer and $1.25 \mathrm{U}$ Taq DNA polymerase. The PCR products were separated by gel electrophoresis for purification and direct sequencing using ABI PRISM 3730XL sequencer (Applied Biosystems, USA). The study was performed according to the Declaration of Helsinki and approved by the Ethics Committees of Affiliated Shengjing Hospital of China Medical University and Affiliated Hospital of Guiyang Medical College. Informed consent was obtained from the patients' parents.

\section{RESULTS}

We searched for deletions in SMN1 exons 7 and 8 (Table1) or subtle mutations of $S M N$ in 113 SMA patients of Chinese origin. Homozygous absence of SMN1 exon 7 was found in $90.3 \%$ $(102 / 113)$ of patients, while homozygous deletion of $S M N 1$ exon 8 with existence of exon 7 was found in 1 type III patient $(0.9 \%$ ), and thus, the rate of deletion of $S M N 1$ exon 7 and/or 8 was $91.2 \%$ $(103 / 113)$ in Chinese SMA patients. Frequency of homozygous deletion of SMN1 exon 7 in type I, II and III were 97.9, 94.7 and 70.4\%, respectively. Both exons 7 and 8 were involved in SMN1 gene deletion in type I and II in $97.9 \%$ and $84.2 \%$, respectively, whereas $37.0 \%$ in type III (Table 1).

\begin{tabular}{|c|c|c|c|c|c|c|}
\hline & $\mathrm{N}$ & $\begin{array}{l}\text { E7 deletion } \\
(\%)\end{array}$ & $\begin{array}{l}\text { Deletion of E7 } \\
\text { and E8 (\%) }\end{array}$ & $\begin{array}{l}\text { E7 deletion and } \\
\text { E8 retention }(\%)\end{array}$ & $\begin{array}{l}\text { E7 retention and } \\
\text { E8 deletion (\%) }\end{array}$ & $\begin{array}{c}\text { Retention of E7 } \\
\text { and E8 (\%) }\end{array}$ \\
\hline Type I & 48 & $47(97.9)$ & 47 (97.9) & $0(0)$ & $0(0)$ & $1(2.1)$ \\
\hline Type II & 38 & $36(94.7)$ & $32(84.2)$ & $4(10.5)$ & $0(0)$ & $2(5.3)$ \\
\hline Type III & 27 & $19(70.4)$ & $10(37.0)$ & $9(33.3)$ & $1(3.7)$ & $7(25.9)$ \\
\hline Total & 113 & $102(90.3)$ & $89(78.8)$ & $13(11.5)$ & $1(0.9)$ & $10(8.9)$ \\
\hline
\end{tabular}

$\mathrm{E}=$ exon.

Ten patients with retention of exons 7 and 8 of $S M N 1$ were analyzed to identify potential compound heterozygotes by sequencing of exons 7 and 8 of $S M N, 2$ (1 type I, 1 type III) of whom showed heterozygous deletion of the $S M N 1$ gene. Genomic sequencing was carried out in the 2 patients, and heterozygous deletions in exons 7 and 8 and subtle mutations in the type I and III SMA patients were shown, respectively (Figure $1 \mathrm{~A}-\mathrm{F}$ ), because $+6 \mathrm{C}_{S M N 1}$ base of exon 7 , $+245 \mathrm{G}_{S M N 1}$ of exon 8, respectively, existed but the peak height was obviously lower than that of $\mathrm{T}_{S M N 2}$ of exon $7, \mathrm{~A}_{S M N 2}$ of exon 8 by sequencing of exons 7 and 8 of $S M N$ (Figure 1A,B,D,E). The patient with 2 SMN1 copies was regarded as not having SMA, and the 2 patients were presumed to 
have a $S M N 1$ copy. By genomic sequencing in the 2 patients with heterozygous deletion of $S M N 1$ gene, we detected the same subtle mutation (c.683 T>A, p.Leu228X) that was located in exon 5 of $S M N$ (Figure 1C,F). Because abnormities of SMN2 were detected in normal individuals, we presumed the mutation was located in $S M N 1$. The mutation had been previously identified in 1 type I or II, 2 type I and 1 type II patients (Tsai et al., 2001; Zeng et al., 2011; Qu and Li et al., 2012), but the mutation with SMA type III has been detected to date only in our SMA patients. Sequencing peak height of exons 7 and 8 of $S M N$ showed $+6 \mathrm{C}_{S M N 1}: \mathrm{T}_{S M N 2}$ in exon $7 \approx 1: 2,+245 \mathrm{G}_{S M N 1}: \mathrm{A}_{S M N 2}$ in exon8 $\approx 1: 2, \mathrm{~A}_{\text {mutation } S M N 1}: \mathrm{T}_{\text {wild } S M N 2}$ in exon $5 \approx 1: 2$ in the SMA type I patient; $+6 \mathrm{C}_{S M N 1}: \mathrm{T}_{S M N 2}$ in exon $7 \approx 1: 3,+245 \mathrm{G}_{S M N 1}: \mathrm{A}_{S M N 2}$ in exon $8 \approx 1: 4, \mathrm{~A}_{\text {mutation } S M N 1}: \mathrm{T}_{\text {wild } S M N 2}$ in exon $5 \approx 1: 3$ in the SMA type III patient (Figure 1A-F). The other 8 patients with homologous retention of exons 7 and 8 did not show detectable subtle mutation by sequencing.
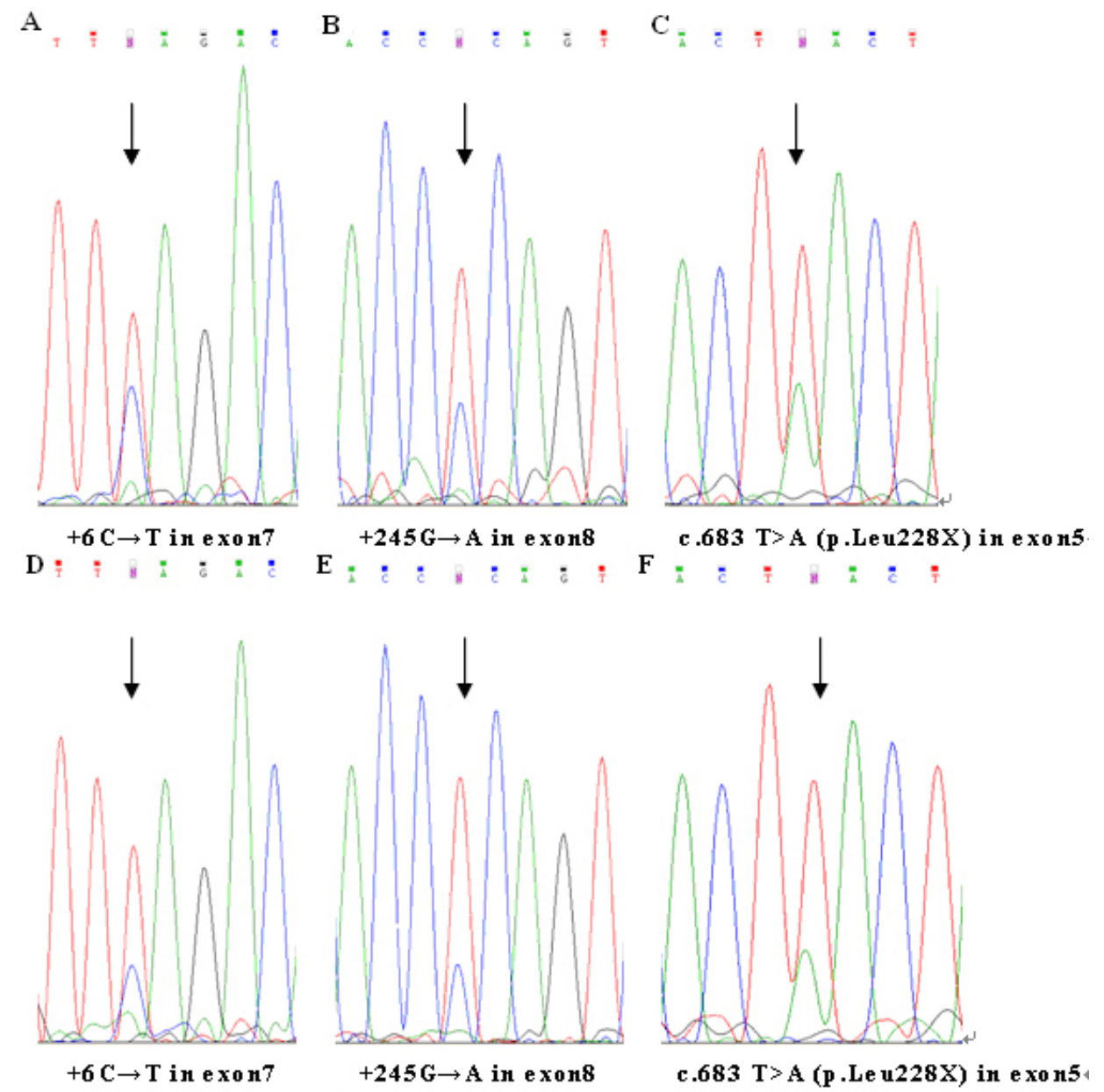

Figure 1. A. D. Direct sequencing showing $+6 \mathrm{C} \rightarrow \mathrm{T}$ in exon7 between $\mathrm{SMN} 1$ and $\mathrm{SMN} 2$. B. E. Reverse sequencing showing $+245 \mathrm{G} \rightarrow \mathrm{A}$ in exon8 between SMN1 and SMN2. C. F. Direct sequencing showing heterozygous SMN1 deletion and c.683 T>A (p.Leu228X) mutations in exon5. A. B. C. = type I SMA patient. D. E. F. = type III SMA patient. 
Clinical characteristics of the 2 patients with compound heterozygous mutations were as follows.

The SMA type I patient died at 4.5 years of age from severe pneumonia. He was born at term (weight $3600 \mathrm{~g}$ ) following a pregnancy of normal duration. He was born to non-consanguineous parents. His elder brother was unable to sit all the time, and died at 4 years of age from severe pneumonia. His elder sister was 14 years old with normal phenotypes. The family noted his decrement in motor functions and generalized muscle weakness after he was 100 days old. He could not control his head and sit alone. His muscle strength in the upper limbs was as low as grade III, and in the lower limbs as low as grade II. He had abolition of all tendon reflex. Electromyography (EMG) showed neurogenic injuries. The result of cranial magnetic resonance imaging (MRI) was normal.

The SMA type III patient was a 9.5-year-old Chinese boy. He was born at term (weight $2900 \mathrm{~g}$ ) following a pregnancy of normal duration. He was born to non-consanguineous parents and was the only child of the family. He could walk unaided at 1 year of age. After 3 years of age, he walked unstably, was able to squat, but could not stand up independently. His muscle strength in the upper limbs was normal, and in lower limbs was grade IV-V. He had abolition of all tendon reflex. EMG showed neurogenic injuries.

The results of subtle mutations of $S M N 1$ of Chinese SMA patients in previously reported subtle mutations and our study are shown in Table 2.

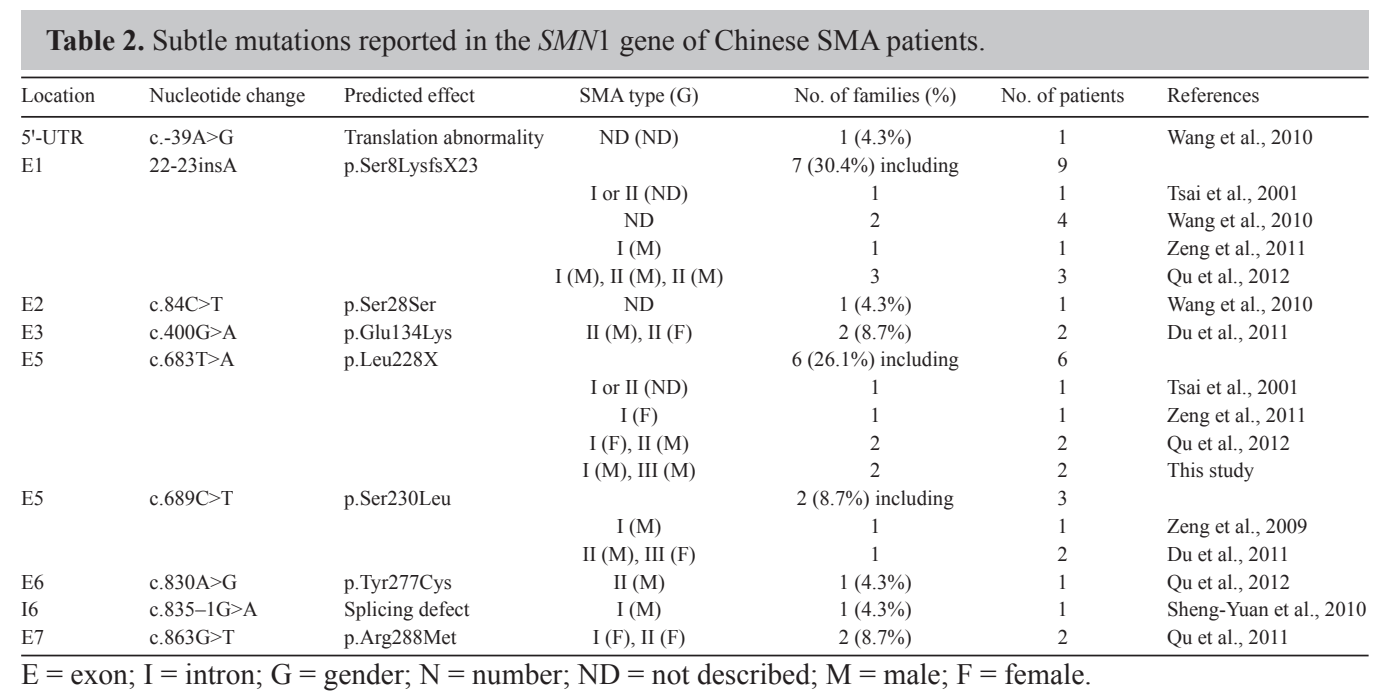

\section{DISCUSSION}

In this study, we analyzed the molecular basis of SMA in a large series of 113 Chinese patients. We found that the rate of homozygous deletion of $S M N 1$ exon7 and/or 8 was $91.2 \%$ $(103 / 113)$ in Chinese SMA patients. The frequency is the same as the approximately $90 \%$ of SMA patients of European origin (Lefebvre et al., 1995; Wirth et al., 2000; Alías et al., 2009). There was a slightly higher frequency of homozygous deletion of SMN1 exon 7 in type I and II (97.9 and $94.7 \%$, respectively) than in type III (70.4\%). The majority of type I and II patients have large deletions of $S M N 1$ exons 7 and 8, whereas some type III patients have deletion 
of $S M N 1$ exons 7 only. Homozygous deletion of $S M N 1$ exon 8 with existence of exon 7 was found in 1 type III patient, and we suspect that deletion of $S M N 1$ exon 8 only may lead to SMA, the result is similar as in a previous study by Ogino et al. (2004).

We found the p.Leu228X mutation was in the 2 patients (1.8\%), where the rate of subtle mutation was $1.8 \%(2 / 113)$ in Chinese SMA patients here. We summarized subtle mutation findings of $S M N 1$ of Chinese SMA patients from previous reported subtle mutations and our study; 9 type subtle mutations in the $S M N 1$ gene have been reported up to now in 26 compound heterozygous Chinese patients from 23 families (Table 2). There are 7 families with missense, 7 frameshift , 6 nonsense, 1 silent, 1 5'-UTR and 1 splice-site mutations in the 23 families. Subtle mutations were detected mainly in exons 1, 5, 3 and 7 of SMN1 gene in Chinese SMA. It is interesting to note that the p.Ser8LysfsX23 and p.Leu228X mutations in exons 1 and 5 seem to be the most common subtle mutations that cause SMA in China, because 13 of 23 families detected had the 2 mutations. The p.Ser8LysfsX23 and p.Leu228X mutations account for 56.5\% (13/23) of the families with subtle mutations reported in the $S M N 1$ gene, which are to date unique to the Chinese population. We revealed 2 subtle mutation hotspots (p.Ser8LysfsX23 and p.Leu228X), which are located in exons 1 and 5 of the $S M N 1$ gene in Chinese patients of SMA firstly. The SMN1 subtle mutations including c.-39A $>$ G, p.Ser8LysfsX23, p.Ser28Ser, p.Leu228X, p.Tyr277Cys and c.835-1G $>$ A were found only in China, and p.Arg288Met was found only in China and Korea (Kang et al., 2009) of Asians to date. We presume the subtle mutation hotspots (p.Ser8LysfsX23 and p.Leu228X) were specific to Chinese. The three most frequently reported subtle mutations of SMA in the United States and Europe are the Y272C missense mutation in exon 6, 768_778dupTGCTGATGCTT frameshift mutation in exon 6 and the 399_402delAGAG frameshift mutation in exon 3 (Ogino et al., 2004). We did not find any mutation in the above 3 most frequently reported subtle mutations in Chinese SMA. It is proposed that the distribution of subtle mutations of $S M N 1$ of SMA is associated with ethnicity or geographic origin.

RNA samples from the patients without deletion of $S M N 1$ gene were not available to detect $S M N 1$ subtle mutation. We analyzed the variation of the sequencing peak height of the two different base pairs of exons 7 and 8 between $S M N 1$ and $S M N 2$ to identify heterozygous deletion of $S M N 1$ gene, and detected subtle mutation by genomic sequencing. We found the same subtle mutation (p.Leu228X) of $S M N 1$ in 2 Chinese SMA patients who showed typical type I and III SMA phenotypes, respectively. This is the first report that the phenotype of SMA type III is associated with $p$.Leu228X mutation worldwide. The ratio of sequencing peak height of the two different base pairs of exons 7 and 8 between $S M N 2$ and $S M N 1$ in the type I SMA patient reported was much lower than that observed in the type III SMA. We presume that the type I SMA patient had 2 SMN2 copies, and that the type III SMA patient had 3 4 SMN2 copies. The more severe the disease is, the less $S M N 2$ copies the patient has (Feldkötter et al., 2002). Our study confirmed the result in the above previous study. This shows that the mutation p.Leu228X is a severe mutation by analysis of the number of $S M N 2$ copies in the 2 patients reported. There are some possible reasons to explain why SMN1 mutations were not identified in the other 8 patients with homologous retention of exons 7 and 8: 1) the unsequenced regions were not analyzed extensively; 2) method of screening analysis was limited; and 3) the patients were considered to have SMN1-unrelated SMA.

\section{ACKNOWLEDGMENTS}

Research supported by the Section of Scientific Research, Science and Technology, 
Department of Guizhou Province, China [(\#2010)3173], the Key Project of the Chinese Ministry of Education (\#212155) and the Science and Technology Fund Project of Department of Health of Guizhou Province (\#gzwkj2012-1-041). The authors thank the patients and their families and the clinicians who collaborated in this study.

\section{REFERENCES}

Alías L, Bernal S, Fuentes-Prior P, Barcelo MJ, et al. (2009). Mutation update of spinal muscular atrophy in Spain: molecular characterization of 745 unrelated patients and identification of four novel mutations in the SMN1 gene. Hum. Genet. 125: 29-39.

Cusin V, Clermont O, Gerard B, Chantereau D, et al. (2003). Prevalence of SMN1 deletion and duplication in carrier and normal populations: implication for genetic counselling. J. Med. Genet. 40: e39.

Du J, Qu YJ, Xiong H, Li EZ, et al. (2011). Mutation analysis of SMN1 gene in patients with spinal muscular atrophy. Zhonghua Er. Ke. Za Zhi. 49: 411-415.

Feldkötter M, Schwarzer V, Wirth R, Wienker TF, et al. (2002). Quantitative analyses of SMN1 and SMN2 based on realtime lightCycler PCR: fast and highly reliable carrier testing and prediction of severity of spinal muscular atrophy. Am. J. Hum. Genet. 70: 358-368.

Kang SH, Cho SI, Chae JH, Chung KN, et al. (2009). False homozygous deletions of SMN1 exon 7 using Dra I PCR-RFLP caused by a novel mutation in spinal muscular atrophy. Genet. Test. Mol. Biomarkers 13: 511-513.

Lefebvre S, Burglen L, Reboullet S, Clermont O, et al. (1995). Identification and characterization of a spinal muscular atrophy-determining gene. Cell 80: 155-165.

Ogino S and Wilson RB (2004). Spinal muscular atrophy: molecular genetics and diagnostics. Expert. Rev. Mol. Diagn. 4: $15-29$.

Parsons DW, McAndrew PE, Iannaccone ST, Mendell JR, et al. (1998). Intragenic telSMN mutations: frequency, distribution, evidence of a founder effect, and modification of the spinal muscular atrophy phenotype by cenSMN copy number. Am. J. Hum. Genet. 63: 1712-1723.

Qu YJ, Song F, Yang YL, Jin YW, et al. (2011). Compound heterozygous mutation in two unrelated cases of Chinese spinal muscular atrophy patients. Chin. Med. J. 124: 385-389.

Qu YJ, Li EZ, Du J, Bai JL, et al. (2012). Subtle mutations in the SMN1 gene in Chinese patients with SMA: p.Arg288Met mutation causing SMN1 transcript exclusion of exon7. BMC Med. Genet. 13: 86.

Sheng-Yuan Z, Xiong F, Chen YJ, Yan TZ, et al. (2010). Molecular characterization of $S M N$ copy number derived from carrier screening and from core families with SMA in a Chinese population. Eur. J. Hum. Genet. 18: 978-984.

Tsai CH, Jong YJ, Hu CJ, Chen CM, et al. (2001). Molecular analysis of SMN, NAIP and P44 genes of SMA patients and their families. J. Neurol. Sci. 190: 35-40.

van der Steege G, Grootscholten PM, van d, V, Draaijers TG, et al. (1995). PCR-based DNA test to confirm clinical diagnosis of autosomal recessive spinal muscular atrophy. Lancet 345: 985-986.

Wang CC, Chang JG, Chen YL, Jong YJ, et al. (2010). Multi-exon genotyping of SMN gene in spinal muscular atrophy by universal fluorescent PCR and capillary electrophoresis. Electrophoresis 31: 2396-2404.

Wirth B (2000). An update of the mutation spectrum of the survival motor neuron gene ( $S M N 1)$ in autosomal recessive spinal muscular atrophy (SMA). Hum. Mutat. 15: 228-237.

Zeng J, Lin YH, Yan AZ, Cai MY, et al. (2009). Mutation analysis of $S M N$ gene in a patient and his family with spinal muscular atrophy. Zhonghua Yi. Xue. Yi. Chuan Xue. Za Zhi. 26: 139-143.

Zeng J, Lin Y, Yan A, Ke L, et al. (2011). Establishment of a molecular diagnostic system for spinal muscular atrophy experience from a clinical laboratory in china. J. Mol. Diagn. 13: 41-47.

Zerres K and Davies KE (1999). 59th ENMC International Workshop: Spinal Muscular Atrophies: recent progress and revised diagnostic criteria 17-19 April 1998, Soestduinen, The Netherlands. Neuromuscul. Disord. 9: 272-278. 